\title{
Weihrauch Degrees, Omniscience Principles and Weak Computability ${ }^{\star}$
}

\author{
Vasco Brattka ${ }^{1}$ and Guido Gherardi ${ }^{2}$ \\ 1 Laboratory of Foundational Aspects of Computer Science, Department of \\ Mathematics \& Applied Mathematics, University of Cape Town, South Africa \\ Vasco.Brattka@uct.ac.za \\ 2 Dipartimento di Filosofia, Università di Bologna, Italy \\ Guido.Gherardi@unibo.it
}

\begin{abstract}
In this paper we study a reducibility that has been introduced by Klaus Weihrauch or, more precisely, a natural extension of this reducibility for multi-valued functions on represented spaces. We call the corresponding equivalence classes Weihrauch degrees and we show that the corresponding partial order induces a lower semi-lattice with the disjoint union of multi-valued functions as greatest lower bound operation. We show that parallelization is a closure operator for this semilattice and the parallelized Weihrauch degrees even form a lattice with the product of multi-valued functions as greatest lower bound operation. We show that the Medvedev lattice and hence the Turing upper semi-lattice can both be embedded into the parallelized Weihrauch lattice in a natural way. The importance of Weihrauch degrees is based on the fact that multi-valued functions on represented spaces can be considered as realizers of mathematical theorems in a very natural way and studying the Weihrauch reductions between theorems in this sense means to ask which theorems can be transformed continuously or computably into each other. This allows a new purely topological or computational approach to metamathematics that sheds new light on the nature of theorems. As crucial corner points of this classification scheme we study the limited principle of omniscience LPO, the lesser limited principle of omniscience LLPO and their parallelizations. We show that parallelized LLPO is equivalent to Weak König's Lemma and hence to the Hahn-Banach Theorem in this new and very strong sense. We call a multi-valued function weakly computable if it is reducible to the Weihrauch degree of parallelized LLPO and we present a new proof that the class of weakly computable operations is closed under composition. This proof is based on a computational version of Kleene's ternary logic. Moreover, we characterize weakly computable operations on computable metric spaces as operations that admit upper semi-computable compact-valued selectors and we show that any single-valued weakly computable operation is already computable in the ordinary sense.
\end{abstract}

* This project has been supported by the Italian Ministero degli Affari Esteri and the National Research Foundation of South Africa (NRF)

Andrej Bauer, Peter Hertling, Ker-I Ko (Eds.)

6th Int'l Conf. on Computability and Complexity in Analysis, 2009, pp. 83-94

http://drops.dagstuhl.de/opus/volltexte/2009/2261 


\section{Introduction}

The purpose of this paper is to propose a new computational approach to metamathematics that is based on the classification of mathematical theorems according to their computational content. Such an approach started with a classification of the Weihrauch degree of the Hahn-Banach Theorem in [1] and the intention here is to lay some careful foundations for further studies. In a following paper [2] we analyze certain choice principles and we present a case study with a classification on many theorems from analysis. This paper is only an extended abstract, but a full version with all definitions and proofs is available for the interested reader [3].

Essentially, the idea is to ask which theorems can be continuously or even computably transferred into each other. In order to give a meaningful interpretation to this idea we consider mathematical theorems as multi-valued operations $F: X \rightrightarrows Y$ that map certain input data $X$ into certain output data $Y$. Such a perspective is very natural, since many theorems in mathematics are actually $\Pi_{2}$ theorems, hence they have the logical form

$$
(\forall x \in X)(\exists y \in Y)(x, y) \in A
$$

and one can just consider $F: X \rightrightarrows Y$ as a realizer or multi-valued Skolem function for this statement.

The appropriate technical tool to study whether two such potentially partial multi-valued functions $F: \subseteq X \rightrightarrows Y$ and $G: \subseteq X \rightrightarrows Y$ can be continuously or computably transferred into each other is Weihrauch reducibility. This is a reducibility that has been introduced by Klaus Weihrauch around 1990 in two unpublished papers $[4,5]$ and since then it has been studied by several others (see for instance $[6-11,1,2,12]$ ).

Originally, this reducibility has been introduced for single-valued functions on Baire space. Basically, the idea is to say that $F$ is strongly Weihrauch reducible to $G$, in symbols $F \leq_{\mathrm{sW}} G$, if there are computable (or alternatively continuous) functions $H$ and $K$ such that

$$
F=H \circ G \circ K
$$

Thus, $K$ acts as an input modification and $H$ acts as an output modification. We will mainly consider the computable version of this reduction here since the positive reduction results are stronger. For negative results the topological version of the reduction is stronger and indeed reductions typically fail for continuity reasons. However, such topological results can usually be derived from computational results by relativization.

It turns out that the strong version of Weihrauch reducibility is slightly too strong for many purposes, since it distinguishes too many functions. For instance the identity cannot be reduced to a constant function in this way, since there is no way to feed the input through a constant function. This is the reason why the more important reducibility is the one where we say that $F$ is Weihrauch 
reducible to $G$, in symbols $F \leq_{\mathrm{W}} G$, if there are computable functions $H$ and $K$ such that

$$
F=H \circ\langle\mathrm{id}, G \circ K\rangle \text {. }
$$

Thus, the difference is that the input is fed through to the outer function $H$ independently of $G$.

Weihrauch $[4,5]$ has already studied an extended version of his reducibility to sets $\mathcal{F}$ and $\mathcal{G}$ of functions on Baire space and $\mathcal{F}$ is called Weihrauch reducible to $\mathcal{G}$, in symbols $\mathcal{F} \leq_{\mathrm{W}} \mathcal{G}$, if there are computable functions $H$ and $K$ such that

$$
(\forall G \in \mathcal{G})(\exists F \in \mathcal{F}) F=H\langle\text { id }, G K\rangle .
$$

Here \langle\rangle$: \mathbb{N}^{\mathbb{N}} \times \mathbb{N}^{\mathbb{N}} \rightarrow \mathbb{N}^{\mathbb{N}}$ denotes a computable standard pairing function [13]. That is, any function $G \in \mathcal{G}$ computes some function $F \in \mathcal{F}$ and the computation is performed uniformly with two fixed computable $H$ and $K$. This extension of Weihrauch reducibility is related to ordinary Weihrauch reducibility exactly as Medvedev reducibility is related to Turing reducibility.

We use this concept to extend Weihrauch reducibility even further to multivalued operations $f: \subseteq X \rightrightarrows Y$ on represented spaces $X$ and $Y$. Roughly speaking, such an $f$ is Weihrauch reducible to an analogous $g$, in symbols $f \leq_{\mathrm{W}} g$, if the set of realizers of $f$ is reducible to the set of realizers of $g$ in the above mentioned sense of Weihrauch reducibility for sets, i.e.

$$
\{F: F \vdash f\} \leq_{\mathrm{W}}\{G: G \vdash g\} .
$$

Here a single-valued $F$ on Baire space is called a realizer of $f$, in symbols $F \vdash f$, if $F$ computes a name $F(p)$ of some output value in $f(x)$, given some name $p$ of $x$. This generalization of Weihrauch reducibility was introduced for single-valued functions in [10] and for multi-valued functions in [1]. We call the corresponding equivalence classes Weihrauch degrees.

Compared to strong Weihrauch reducibility, the ordinary version of Weihrauch reducibility has exactly the right degree of precision, it distinguishes exactly what should be distinguished computationally, but not more. Among all functions (with at least one computable point in the domain) the computable ones form the least degree. For the continuous version of Weihrauch reducibility exactly the continuous functions form the least degree (among all functions with non-empty domain).

\section{Products, sums and parallelization}

In this section we briefly summarize some of our results on some basic properties of Weihrauch reducibility and of Weihrauch degrees. In particular we investigate the product operation $f \times g$ and the direct sum $f \oplus g$ of multi-valued operations and we show that both operations are monotone with respect to Weihrauch reducibility. While the product preserves single-valuedness, the disjoint union does not and hence it requires multi-valuedness in order to be meaningful. Among other things the partial order on Weihrauch degrees induces a lower semi-lattice 
with direct sums as greatest lower bounds. While the product operation is just the ordinary product operation of multi-valued functions, we define the direct sum as follows. For any two sets $Y, Z$ we define the direct sum or disjoint union by $Y \oplus Z:=(\{0\} \times Y) \cup(\{1\} \times Z)$.

Definition 1 (Direct sum). Let $f: \subseteq X \rightrightarrows Y$ and $g: \subseteq U \rightrightarrows V$ be multivalued maps on represented spaces. Then the direct sum of these maps $f \oplus g: \subseteq$ $X \times U \rightrightarrows Y \oplus V$ is defined by $(f \oplus g)(x, u):=(\{0\} \times f(x)) \cup(\{1\} \times g(u))$ for all $(x, u) \in \operatorname{dom}(f \oplus g):=\operatorname{dom}(f) \times \operatorname{dom}(g)$.

The first observation is that product and sum are both monotone operations in the sense that

$$
-f \leq_{\mathrm{W}} g \text { and } f^{\prime} \leq_{\mathrm{W}} g^{\prime} \Longrightarrow f \times f^{\prime} \leq_{\mathrm{W}} g \times g^{\prime} \text { and } f \oplus f^{\prime} \leq_{\mathrm{W}} g \oplus g^{\prime} .
$$

This monotonicity result guarantees that we can safely extend the product and direct sum operation to Weihrauch degrees. Other common properties of products and sums are that they are both associative and commutative on degrees. The identity is a neutral element with respect to products. An important difference between product and sum is that functions are not necessarily idempotent with respect to products, i.e. there are $f$ such that $f \not_{\mathrm{W}} f \times f$, while idempotency is always given for sums. A crucial property of sums is that they yield the greatest lower bound with respect to Weihrauch reducibility.

Proposition 1 (Greatest lower bound). Let $f$ and $g$ be multi-valued functions on represented spaces. Then $f \oplus g$ is the greatest lower bound of $f$ and $g$ with respect to Weihrauch reducibility $\leq_{\mathrm{W}}$ and strong Weihrauch reducibility $\leq_{\mathrm{sW}}$.

Is there any multi-valued map that plays the role of a neutral element with respect to the sum operation? Naturally, this would have to be a multi-valued function with an empty set of realizers. One should note that this is not the nowhere defined function $f: \subseteq X \rightrightarrows Y$, since $\{F: F \vdash f\}$ is the set of all function $F: \subseteq \mathbb{N}^{\mathbb{N}} \rightarrow \mathbb{N}^{\mathbb{N}}$. If we accept the Axiom of Choice, then clearly, a function without realizers does not exist and hence we add an extra object 0 to our structure with $\{F: F \vdash 0\}=\emptyset$. Weihrauch reducibility can straightforwardly be extended to multi-valued functions enriched by 0 , just by using $\emptyset$ as the set of realizers of 0 . We denote the Weihrauch degree of 0 by $\mathbf{0}$. We assume that we have a fixed underlying set of represented spaces $\mathcal{R}$ and we also assume that this set includes $\left(\mathbb{N}^{\mathbb{N}}\right.$, id) and that $\mathcal{R}$ is closed under products and direct sums.

Definition 2 (Set of Weihrauch degrees). Let $\mathcal{W}$ denote the set that contains the degree $\mathbf{0}$ and all Weihrauch degrees of all multi-valued operations $f: \subseteq X \rightrightarrows Y$ with at least one computable point in $\operatorname{dom}(f)$ and with represented spaces $X, Y \in \mathcal{R}$. By 1 we denote the degree of the computable functions in $\mathcal{W}$.

In the following theorem we collect all the structural properties of Weihrauch degrees that we have studied so far. 
Theorem 1 (Weihrauch degrees). The space $\left(\mathcal{W}, \leq_{\mathrm{W}}\right)$ of Weihrauch degrees is a lower semi-lattice with least element $\mathbf{1}$ and greatest element $\mathbf{0}$ and with $\oplus$ as the greatest lower bound operation. In particular, $(\mathcal{W}, \oplus)$ is an idempotent monoid with neutral element $\mathbf{0}$. Moreover, $(\mathcal{W}, \times)$ is a monoid with neutral element 1.

An important operation on functions is parallelization $\widehat{f}$, which means to take countably many copies of the function $f$ in parallel, i.e.

$$
\widehat{f}\left(x_{0}, x_{1}, x_{2}, \ldots\right):=f\left(x_{0}\right) \times f\left(x_{1}\right) \times f\left(x_{2}\right) \times \ldots
$$

This operation forms a closure operator with respect to Weihrauch reducibility.

Proposition 2 (Parallelization). Let $f$ and $g$ be multi-valued functions on represented spaces. Then

1. $f \leq_{\mathrm{W}} \widehat{f}$

2. $f \leq_{\mathrm{W}} g \Longrightarrow \widehat{f} \leq_{\mathrm{W}} \widehat{g}$

(extensive)

3. $\widehat{f} \equiv_{\mathrm{W}} \widehat{\hat{f}}$

(increasing)

(idempotent)

An analogous result holds for strong Weihrauch reducibility.

The fact that Weihrauch reducibility is a closure operator allows us to define a parallelized version of Weihrauch reducibility.

Definition 3 (Parallel reducibility). Let $f$ and $g$ be multi-valued operations on represented spaces. Then we say that $f$ is parallely Weihrauch reducible to $g$, in symbols $f \leq_{\widehat{\mathrm{W}}} g$, if $\widehat{f} \leq_{\mathrm{W}} \widehat{g}$. We say that $f$ is parallely Weihrauch equivalent to $g$, in symbols $f \equiv_{\widehat{\mathrm{W}}} g$, if $f \leq_{\widehat{\mathrm{W}}} g$ and $g \leq_{\widehat{\mathrm{W}}} f$ holds. We call the corresponding equivalence classes parallel Weihrauch degrees.

Parallel reducibility is compatible with products and sums in the following sense:

$-\widehat{f \times g} \equiv_{\mathrm{sW}} \widehat{f} \times \widehat{g}$ and $\widehat{f \oplus g} \leq_{\mathrm{sW}} \widehat{\hat{f} \oplus \widehat{g}} \equiv_{\mathrm{sW}} \widehat{f} \oplus \widehat{g}$.

Moreover, parallel Weihrauch degrees are idempotent with respect to products, i.e. $\widehat{f} \equiv_{\mathrm{sW}} \widehat{f} \times \widehat{f}$. The idempotency of parallel Weihrauch degrees has the consequence that the product actually is the least upper bound operation for parallel Weihrauch degrees ${ }^{3}$.

Proposition 3 (Least upper bound). Let $f$ and $g$ be multi-valued functions on represented spaces. Then $f \times g$ is the least upper bound of $f$ and $g$ with respect to parallel Weihrauch reducibility $\leq_{\widehat{\mathrm{W}}}$.

\footnotetext{
${ }^{3}$ Independently, Arno Pauly [12] has recently proved that another operation on functions that takes direct sums on the input and output side yields a supremum even in the non-parallelized case. He has also proved that the corresponding upper semilattice is distributive.
} 
The parallelized Weihrauch degrees together with their partial order even form a lattice with the product as least upper bound operation. By $\widehat{\mathcal{W}}$ we denote the set of parallel Weihrauch degrees, which is defined as $\mathcal{W}$ but using parallel Weihrauch reducibility. As a corollary of our results we obtain that the parallel Weihrauch degrees of multi-valued functions form a lattice.

Theorem 2 (Parallel Weihrauch degrees). The space $\left(\widehat{\mathcal{W}}, \leq_{\widehat{\mathrm{W}}}\right)$ of parallel Weihrauch degrees is a lattice with least element $\mathbf{1}$ and greatest element $\mathbf{0}$, with $\oplus$ as the greatest lower bound operation and with $\times$ as the least upper bound operation. In particular, $(\widehat{\mathcal{W}}, \oplus)$ and $(\widehat{\mathcal{W}}, \times)$ are idempotent monoids with neutral elements $\mathbf{0}$ and $\mathbf{1}$, respectively.

\section{Embedding of Turing degrees and Medvedev degrees}

Now we mention that the Medvedev lattice can be embedded into the Weihrauch lattice such that least upper bounds and greatest lower bounds are preserved. This embedding only requires total and continuous multi-valued operations on Baire space. As a consequence, we obtain that Turing degrees can be embedded such that least upper bounds are preserved and this embedding only requires total and continuous single-valued functions on Baire space.

We recall that a set $\mathcal{A} \subseteq \mathbb{N}^{\mathbb{N}}$ is said to be Medvedev reducible to $\mathcal{B} \subseteq \mathbb{N}^{\mathbb{N}}$, in symbols $\mathcal{A} \leq_{\mathrm{M}} \mathcal{B}$, if there exists a computable $F: \subseteq \mathbb{N}^{\mathbb{N}} \rightarrow \mathbb{N}^{\mathbb{N}}$ with $\mathcal{B} \subseteq \operatorname{dom}(F)$ and $F(\mathcal{B}) \subseteq \mathcal{A}$. In fact, Turing reducibility is a special case, since $p \in \mathbb{N}^{\mathbb{N}}$ is said to be Turing reducible to $q \in \mathbb{N}^{\mathbb{N}}$, in symbols $p \leq_{\mathrm{T}} q$, if $\{p\} \leq_{\mathrm{M}}\{q\}$ (see [14]).

Now we associate to any $q \in \mathbb{N}^{\mathbb{N}}$ the constant function $c_{q}: \mathbb{N}^{\mathbb{N}} \rightarrow \mathbb{N}^{\mathbb{N}}, p \mapsto q$ for all $p \in \mathbb{N}^{\mathbb{N}}$. In the next step we associate a multi-valued function to any non-empty $\mathcal{A} \subseteq \mathbb{N}^{\mathbb{N}}$ by $c_{\mathcal{A}}: \mathbb{N}^{\mathbb{N}} \rightrightarrows \mathbb{N}^{\mathbb{N}}, p \mapsto \mathcal{A}$ for all $p \in \mathbb{N}^{\mathbb{N}}$. Then $c_{\mathcal{A}}$ has a computable realizer if and only if $\mathcal{A}$ contains a computable member. To the empty set $\emptyset \subseteq \mathbb{N}^{\mathbb{N}}$ we associate $c_{\emptyset}:=0$, the special "multi-valued function" without realizer. We note that the function $c_{\mathcal{A}}$ is parallelizable, i.e. $c_{\mathcal{A}} \equiv_{\mathrm{W}} \widehat{c_{\mathcal{A}}}$. Our main result of this section is now the following theorem.

Theorem 3 (Embedding of Medvedev degrees). Let $\mathcal{A}, \mathcal{B} \subseteq \mathbb{N}^{\mathbb{N}}$. Then $\mathcal{A} \leq_{\mathrm{M}} \mathcal{B} \Longleftrightarrow c_{\mathcal{A}} \leq_{\mathrm{W}} c_{\mathcal{B}}$.

It is clear that a corresponding embedding of Turing degrees follows, i.e. $p \leq_{\mathrm{T}} q \Longleftrightarrow c_{p} \leq_{\mathrm{W}} c_{q}$. Now we want to show that our embedding of the Medvedev lattice preserves also greatest lower and least upper bounds. For sets $\mathcal{A}, \mathcal{B} \subseteq \mathbb{N}^{\mathbb{N}}$ one usually defines $\mathcal{A} \oplus \mathcal{B}:=\{\langle p, q\rangle: p \in \mathcal{A}$ and $q \in \mathcal{B}\}$ and $\mathcal{A} \otimes \mathcal{B}:=0 \mathcal{A} \cup 1 \mathcal{B}$. The reader should note that product and sum are just swapped compared to the way we use these operations. Now one can easily show the following result.

Proposition 4. Let $\mathcal{A}, \mathcal{B} \subseteq \mathbb{N}^{\mathbb{N}}$. Then $c_{\mathcal{A} \oplus \mathcal{B}} \equiv_{\mathrm{sW}} c_{\mathcal{A}} \times c_{\mathcal{B}}$ and $c_{\mathcal{A} \otimes \mathcal{B}} \equiv_{\mathrm{sW}} c_{\mathcal{A}} \oplus c_{\mathcal{B}}$.

We mention that this result implies that our embedding of the Medvedev lattice preserves least upper bounds and greatest lower bounds. 
Corollary 1 (Embedding of the Medvedev lattice). The Medvedev lattice is embeddable into the parallel Weihrauch lattice (restricted to total and continuous multi-valued functions on Baire space and 0) with an embedding that preserves least upper bounds and greatest lower bounds.

We also formulate the analogous result for Turing degrees.

Corollary 2 (Embedding of the Turing upper semi-lattice). The upper semi-lattice of Turing degrees is embeddable into the parallel Weihrauch lattice (restricted to total and continuous single-valued functions on Baire space) with an embedding that preserves least upper bounds.

Using these results some structural properties of the parallel Weihrauch lattice can be transferred from the Turing uppers semi-lattice and the Medvedev lattice. This observation also gives raise to plenty of further research questions.

\section{Omniscience principles}

In this section we study the the limited principle of omniscience LPO and the lesser limited principle of omniscience LLPO in the upper semi-lattice of Weihrauch reducibility. Such a study has also already been initiated by Weihrauch [5]. The principles themselves have originally been introduced by Brouwer and Bishop in constructive mathematics $[15,16]$. Roughly speaking, LPO corresponds to the law of the excluded middle $(A \vee \neg A)$ and LLPO to de Morgan's law $\neg(A \wedge B) \Longleftrightarrow(\neg A \vee \neg B)$, both restricted to simple existential statements. More precisely, they are stated as follows:

Definition 4 (Omniscience principles). We define:

$$
\begin{aligned}
& -\operatorname{LPO}: \mathbb{N}^{\mathbb{N}} \rightarrow \mathbb{N}, \quad \operatorname{LPO}(p)=\left\{\begin{array}{l}
0 \text { if }(\exists n \in \mathbb{N}) p(n)=0, \\
1 \text { otherwise }
\end{array}\right. \\
& -\operatorname{LLPO}: \subseteq \mathbb{N}^{\mathbb{N}} \rightrightarrows \mathbb{N}, \operatorname{LLPO}(p) \ni\left\{\begin{array}{l}
0 \text { if }(\forall n \in \mathbb{N}) p(2 n)=0 \\
1 \text { if }(\forall n \in \mathbb{N}) p(2 n+1)=0
\end{array},\right.
\end{aligned}
$$

where $\operatorname{dom}($ LLPO $):=\left\{p \in \mathbb{N}^{\mathbb{N}}: p(k) \neq 0\right.$ for at most one $\left.k\right\}$.

One should notice that the definition of LLPO implies that $\operatorname{LLPO}\left(0^{\mathbb{N}}\right)=$ $\{0,1\}$. The two principles LPO and LLPO have already been studied in computable analysis [4-7]. For instance, it is well-known that LPO is reducible to any other discontinuous single-valued function on Baire space (see Lemma 8.2.6 in [13]).

Proposition 5. Let $F: \subseteq \mathbb{N}^{\mathbb{N}} \rightarrow \mathbb{N}^{\mathbb{N}}$ be discontinuous. Then we obtain $\mathrm{LPO} \leq_{\mathrm{sW}} F$, relatively to some oracle.

While LPO is the "simplest" single-valued discontinuous function, its parallelization $\widehat{\mathrm{LPO}}$ is at the other end of the spectrum, it is complete among all $\Sigma_{2}^{0}$-measurable functions with respect to the Borel hierarchy. 
Similarly, as $\widehat{\mathrm{LPO}}$ is complete for the class of limit computable operations, we will show that $\widehat{\mathrm{LLPO}}$ is also complete for a very natural class of operations that we will call weakly computable. For technical simplicity by $\widehat{\mathrm{LLPO}}$ we actually mean

$$
\widehat{\mathrm{LLPO}}\left\langle p_{0}, p_{1}, \ldots\right\rangle(k) \ni\left\{\begin{array}{l}
0 \text { if }(\forall n) p_{k}(2 n)=0 \\
1 \text { if }(\forall n) p_{k}(2 n+1)=0
\end{array}\right.
$$

One benefit of this understanding of $\widehat{\mathrm{LLPO}}$ is that it is composable with itself and the next observation is that the composition of $\widehat{\text { LLPO }}$ with itself is strongly below itself. Roughly speaking this is because LLPO is defined only in terms of universal quantifiers and two consecutive universal quantifiers can be absorbed in one.

Lemma 1. $\widehat{\mathrm{LLPO}} \circ \widehat{\mathrm{LLPO}} \leq_{\mathrm{sW}} \widehat{\mathrm{LLPO}}$.

Using the NAND operation and Kleene's ternary logic we can show another interesting property of $\widehat{\mathrm{LLPO}}$, namely that it has some quasi-continuity property although it is discontinuous and we will exploit this property for our main result in this section. This result can also be interpreted as a completeness result for parallelized LLPO.

Theorem 4 (Completeness of parallelized LLPO). For any computable function $F: \subseteq\{0,1\}^{\mathbb{N}} \rightarrow\{0,1\}^{\mathbb{N}}$ there exists a computable function $G: \subseteq\{0,1\}^{\mathbb{N}} \rightarrow$ $\{0,1\}^{\mathbb{N}}$ such that $F \circ \widehat{\mathrm{LLPO}}=\widehat{\mathrm{LLPO}} \circ G$.

If we combine the results showed so far, then we obtain that the multivalued operations below $\widehat{\mathrm{LLPO}}$ are closed under composition. This has first been observed in [1], where it was expressed in terms of Weak König's Lemma (see also Corollary 3).

Proposition 6 (Composition). Let $f: \subseteq X \rightrightarrows Y$ and $g: \subseteq Y \rightrightarrows Z$ be multivalued operations on represented spaces. Then

$$
f \leq_{\mathrm{W}} \widehat{\mathrm{LLPO}} \text { and } g \leq_{\mathrm{W}} \widehat{\mathrm{LLPO}} \Longrightarrow g \circ f \leq_{\mathrm{W}} \widehat{\mathrm{LLPO}} .
$$

The same holds true with respect to some oracle (i.e. we can replace Weihrauch reducibility by its continuous counterpart in all occurrences here).

We believe that this result justifies to give a new name to the operations below $\widehat{\mathrm{LLPO}}$.

Definition 5 (Weakly computable). A function $f: \subseteq X \rightrightarrows Y$ on represented spaces $X, Y$ is called weakly computable, if $f \leq_{\mathrm{W}} \widehat{\mathrm{LLPO}}$. Similarly, such a function is called weakly continuous, if $f \leq_{\mathrm{W}} \widehat{\mathrm{LLPO}}$ holds with respect to some oracle.

One main goal of this section is to show the following theorem on the omniscience principles. This theorem completely characterizes the relation of the omniscience principles and their parallelizations with respect to Weihrauch reducibility. 
Theorem 5 (Omniscience principles). LLPO $<\left._{\mathrm{W}} \mathrm{LPO}\right|_{\mathrm{W}} \widehat{\mathrm{LLPO}}<_{\mathrm{W}} \widehat{\mathrm{LPO}}$. All negative results also hold true with respect to some arbitrary oracle.

Note that the proof even shows the strong reduction LLPO $\leq_{\mathrm{sW}}$ LPO. A different direct proof of LPO $\mathbb{E}_{\mathrm{W}} \mathrm{LLPO}$ is presented in Theorem 4.2 in [5].

Since any discontinuous single-valued function is already above LPO, it is

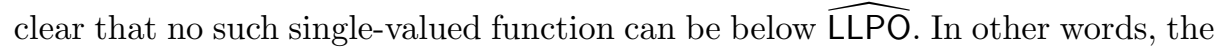
parallel Weihrauch degree of LLPO has no single-valued member. In particular, this means that multi-valuedness does not appear accidentally in our theory, but in some sense it is unavoidable. Indeed we will show in Corollary 4 that any single-valued weakly computable function is already computable in the ordinary sense.

This has surprising algorithmic consequences. Any "algorithm" that uses weakly computable operations such as $x \leq 0$ or $x \geq 0$ leads to a computable result, as long as the result is uniquely determined, i.e. single-valued. And this is so, although these operations are typically discontinuous and non-computable.

\section{Compact choice and Weak König's Lemma}

In this section we will show that the parallel version of LLPO is equivalent to Weak König's Lemma. We first formalize Weak Kőnig's Lemma for this purpose. We recall that a binary tree is a subset $T \subseteq\{0,1\}^{*}$ that is closed under the prefix relation, i.e. if $w \in T$ and $v \sqsubseteq w$, then $v \in T$. We use some standard bijective enumeration $\left(w_{n}\right)_{n \in \mathbb{N}}$ of all the binary words. By $\operatorname{Tr}$ we denote the set of all binary trees and we use a representation $\delta_{\operatorname{Tr}}$ of Tr that is defined by

$$
\delta_{\operatorname{Tr}}(p)=T: \Longleftrightarrow \chi_{T}\left(w_{n}\right)=p(n),
$$

where $\chi_{T}:\{0,1\}^{*} \rightarrow\{0,1\}$ denotes the characteristic function of the binary tree $T$. The classical statement of König's Lemma is that any infinite binary tree has an infinite path. An infinite path of $T$ is a sequence $p \in\{0,1\}^{\mathbb{N}}$, such that $p[n] \in T$ for all $n \in \mathbb{N}$. Here $p[n]=p(0) \ldots p(n-1)$ is the prefix of $p$ of length $n$. By $[T]$ the set of infinite paths of $T$ is denoted. Now we can formalize Weak König's Lemma as follows.

Definition 6 (Weak König's Lemma). We define a multi-valued operation WKL $: \subseteq \operatorname{Tr} \rightrightarrows\{0,1\}^{\mathbb{N}}, T \mapsto[T]$ with $\operatorname{dom}(\mathrm{WKL})=\left\{T \subseteq\{0,1\}^{*}: T\right.$ is an infinite binary tree\}.

Weak König's Lemma has already been studied in this form in [1]. Our main result here is that the parallel version of LLPO is strongly equivalent to Weak König's Lemma. For the proof we use Weak König's Lemma itself.

Theorem 6 (Weak König's Lemma). WKL $\equiv_{\mathrm{sW}} \widehat{\mathrm{LLPO}}$.

In [1] it has been proved that the Hahn-Banach Theorem HBT has the same Weihrauch degree as WKL and hence the same Weihrauch degree as $\widehat{\text { LLPO. We }}$ formulate this as a corollary without exactly specifying HBT (the reader is referred to [1] for details). 
Corollary 3. HBT $\equiv_{W} W K L \equiv_{W} \widehat{L L P O}$.

Another equivalence that has been proved in [1] is that all the aforementioned theorems are equivalent to compact choice in rich spaces. We will use this observation and we adapt the formulation to our context.

Definition 7 (Compact choice). Let $X$ be a computable metric space. The multi-valued operation $\mathrm{C}_{\mathcal{K}(X)}: \subseteq \mathcal{K}_{-}(X) \rightrightarrows X, A \mapsto A$ with $\operatorname{dom}\left(\mathrm{C}_{\mathcal{K}(X)}\right):=$ $\{A \subseteq X: A \neq \emptyset\}$ is called compact choice of $X$.

Here $\mathcal{K}_{-}(X)$ denotes the set of compact subsets of $X$, which is equipped with the negative information representation $\kappa_{-}$(here a name of a compact set $K$ is a list of all finite open rational covers of $K$, see [17] for details). In some sense, WKL is compact choice for the Cantor space $\{0,1\}^{\mathbb{N}}$ and, in fact, in [1] it has been proved that compact choice for a large class of computable metric space is equivalent to $C_{\mathcal{K}\left(\{0,1\}^{\mathrm{N}}\right)} \equiv_{\mathrm{W}} \mathrm{WKL}$. Using this result we mention a slightly different result here adapted to our operations.

Theorem 7 (Compact choice). Let $X$ be a computable metric space. Then $\mathrm{C}_{\mathcal{K}(X)} \leq_{\mathrm{sW}} \widehat{\mathrm{LLPO}}$. If $X$ is rich, i.e. if there is a computable embedding $\iota:\{0,1\}^{\mathbb{N}} \hookrightarrow$ $X$, then $\mathrm{C}_{\mathcal{K}(X)} \equiv_{\mathrm{sW}} \widehat{\mathrm{LLPO}}$.

The characterization of $\widehat{\mathrm{LLPO}}$ as compact choice allows us to derive a characterization of weakly computable operations. Now we are prepared to show the characterization of weakly computable operations. We say that a function $s: \subseteq X \rightarrow \mathcal{K}_{-}(Y)$ is a selector of a function $f: \subseteq X \rightrightarrows Y$, if $\operatorname{dom}(s)=\operatorname{dom}(f)$ and $s(x) \subseteq f(x)$ for all $x \in \operatorname{dom}(f)$. Continuous functions $s: \subseteq X \rightarrow \mathcal{K}_{-}(Y)$ are also called upper semi-continuous.

Theorem 8 (Selection). Let $X$ be a represented space and let $Y$ be a computable metric space. A function $f: \subseteq X \rightrightarrows Y$ is weakly computable if and only if $f$ admits a computable selector $s: \subseteq X \rightarrow \mathcal{K}_{-}(Y)$.

It is known that for computable metric spaces $\left(Y, \delta_{Y}\right)$ the singleton operation $Y \rightarrow \mathcal{K}_{-}(Y), y \mapsto\{y\}$ that maps a point to the corresponding singleton set is $\left(\delta_{Y}, \kappa_{-}\right)$-computable and it admits a $\left(\kappa_{-}, \delta_{Y}\right)$-computable right inverse (see for instance Lemma 6.4 in [18]). Thus we obtain the following corollary of the Selection Theorem 8.

Corollary 4 (Weakly computability). Let $X$ be a represented space and $Y$ a computable metric space. Any weakly computable single-valued operation $f: \subseteq$ $X \rightarrow Y$ is computable.

Similarly, it follows that any weakly continuous single-valued function is already continuous in the ordinary sense. 


\section{Conclusions}

In this paper we have studied Weihrauch reducibility of multi-valued functions on represented spaces. Among other things, we have proved that Weihrauch degrees form a lower semi-lattice with the direct sum operation as greatest lower bound operation. Moreover, we have studied parallelization as closure operator and we have shown that the parallelized Weihrauch degrees even form a lattice with the product as least upper bound operation. The Medvedev lattice and the upper semi-lattice of Turing degrees can be embedded into the parallelized Weihrauch lattice. Moreover, we have proved that the parallelized versions $\widehat{\text { LPO }}$ and $\widehat{\text { LLPO }}$ of the limited principle of omniscience and the lesser limited principle of omniscience, respectively, play a crucial role in our lattice. While $\widehat{\mathrm{LPO}}$ is complete for the class of limit computable operations, we have shown that $\widehat{\text { LLPO }}$ can be used to define a meaningful class of weakly computable operations that is closed under composition. Single-valued weakly computable operations are already computable in the ordinary sense. This fact could be related to conservativeness properties of $\mathrm{WKL}_{0}$ in reverse mathematics $[19,20]$ and to known uniqueness properties in constructive mathematics [21-24].

In a forthcoming paper [2] we discuss the classification of the Weihrauch degree of many theorems from analysis, such as the Intermediate Value Theorem, the Baire Category Theorem, the Banach Inverse Mapping Theorem and many others. It turns out that certain choice principles are crucial cornerstones for that classification and we believe that our classification sheds new light on the computational properties of these theorems. In particular, our classification seems to be in a well-defined sense finer than other known classifications in constructive and reverse mathematics.

\section{References}

1. Gherardi, G., Marcone, A.: How incomputable is the separable Hahn-Banach theorem? Notre Dame Journal of Formal Logic (to appear) extended abstract version in CCA 2008 proceedings ENTCS 221 (2008) 85-102.

2. Brattka, V., Gherardi, G.: Effective choice and boundedness principles in computable analysis. http://arxiv.org/abs/0905.4685 (preliminary version)

3. Brattka, V., Gherardi, G.: Weihrauch degrees, omniscience principles and weak computability. http://arxiv.org/abs/0905.4679 (preliminary version)

4. Weihrauch, K.: The degrees of discontinuity of some translators between representations of the real numbers. Technical Report TR-92-050, International Computer Science Institute, Berkeley (1992)

5. Weihrauch, K.: The TTE-interpretation of three hierarchies of omniscience principles. Informatik Berichte 130, FernUniversität Hagen, Hagen (1992)

6. Stein, T.v.: Vergleich nicht konstruktiv lösbarer Probleme in der Analysis. Diplomarbeit, Fachbereich Informatik, FernUniversität Hagen (1989)

7. Mylatz, U.: Vergleich unstetiger Funktionen in der Analysis. Diplomarbeit, Fachbereich Informatik, FernUniversität Hagen (1992)

8. Hertling, P.: Unstetigkeitsgrade von Funktionen in der effektiven Analysis. Informatik Berichte 208, FernUniversität Hagen, Hagen (1996) Dissertation. 
9. Brattka, V.: Computable invariance. Theoretical Computer Science 210 (1999) $3-20$

10. Brattka, V.: Effective Borel measurability and reducibility of functions. Mathematical Logic Quarterly 51(1) (2005) 19-44

11. Mylatz, U.: Vergleich unstetiger Funktionen: "Principle of Omniscience" und Vollständigkeit in der $C$-hierarchie. PhD Thesis, Faculty for Mathematics and Computer Science, University Hagen, Germany (2006)

12. Pauly, A.: On the (semi)lattices induced by continuous reducibilities. http://arxiv.org/abs/0903.2177 (preliminary version)

13. Weihrauch, K.: Computable Analysis. Springer, Berlin (2000)

14. Rogers, H.: Theory of Recursive Functions and Effective Computability. McGrawHill, New York (1967)

15. Bishop, E., Bridges, D.S.: Constructive Analysis. Volume 279 of Grundlehren der Mathematischen Wissenschaften. Springer, Berlin (1985)

16. Bridges, D., Richman, F.: Varieties of Constructive Mathematics. Volume 97 of London Mathematical Society Lecture Note Series. Cambridge University Press, Cambridge (1987)

17. Brattka, V., Presser, G.: Computability on subsets of metric spaces. Theoretical Computer Science 305 (2003) 43-76

18. Brattka, V.: Plottable real number functions and the computable graph theorem. SIAM Journal on Computing 38(1) (2008) 303-328

19. Simpson, S.G.: Subsystems of Second Order Arithmetic. Perspectives in Mathematical Logic. Springer, Berlin (1999)

20. Simpson, S., Tanaka, K., Yamazaki, T.: Some conservation results on weak König's lemma. Annals of Pure and Applied Logic 118(1-2) (2002) 87-114

21. Kohlenbach, U.: Effective moduli from ineffective uniqueness proofs. an unwinding of de La Vallé Poussin's proof for Chebycheff approximation. Annals of Pure and Applied Logic 64 (1993) 27-94

22. Schuster, P.: Unique solutions. Mathematical Logic Quarterly 52(6) (2006) 534539

23. Schuster, P.: Corrigendum to "unique solutions". Mathematical Logic Quarterly 53(2) (2007) 214-214

24. Ishihara, H.: Unique existence and computability in constructive reverse mathematics. In Cooper, S.B., Löwe, B., Sorbi, A., eds.: Computation and Logic in the Real World. Volume 4497 of Lecture Notes in Computer Science., Berlin, Springer (2007) 368-377 Third Conference on Computability in Europe, CiE 2007, Siena, Italy, June 18-23, 2007. 